\title{
Speciation of Candida using HiCrome Candida Differential Agar
}

\author{
Soumya Kaup $^{1 *}$, Jaya Sankarankutty ${ }^{2}$, H.V. Balasubrahmanya ${ }^{3}$, \\ Suma Kulkarni ${ }^{1}$ and M. Nirmala ${ }^{1}$ \\ ${ }^{1}$ Department of Microbiology, Shridevi Institute of Medical Sciences and Research Hospital, \\ Sira Road, NH-04, Tumkur-572106, India \\ ${ }^{2}$ Department of Hospital Infection Control, Narayana Hrudayalaya Hospital, Bangalore, India \\ ${ }^{3}$ P.C. Institute of Paramedical Sciences, Bangalore, India \\ *Corresponding author
}

\begin{abstract}
A B S T R A C T
Keywords

Candida,

HiCrome agar,

Cornmeal agar,

chromogenic

media.

\section{Article Info}

Accepted:

12 June 2016

Available Online:

10 July 2016

Non-albicans Candida (NAC) species are emerging as important opportunistic pathogens. This has significant clinical impact as, NAC species have decreased susceptibility to commonly used antifungal agents. Hence species identification in the Clinical Microbiology laboratory is essential. This study was conducted to assess efficacy of HiCrome agar to reliably identify Candida to the species level. Altogether 48 isolates of Candida were isolated during a period of one year constituting 24 isolates of Candida albicans, 13 of Candida tropicalis, 4 of Candida krusei, 4 of Candida glabrata, 2 of Candida parapsilosis and 1 isolate of Candida guillermondii. Identification was done based on microscopic morphology, germ tube test, growth at $45^{\circ} \mathrm{C}$, morphology on corn meal agar and colony colour on HiCrome agar. HiCrome agar accurately identified all species of Candida albicans, Candida tropicalis, Candida krusei and Candida glabrata. Two isolates of Candida parapsilosis and one isolate of Candida guillermondii was misidentified as Candida glabrata. HiCrome agar can be used as a fairly reliable and time saving alternative to conventional methods with good sensitivity and specificity.
\end{abstract}

\section{Introduction}

Candida species have emerged as an important cause of community acquired and nosocomial infections in the past decade. The increasing number of candidial infections can be attributed to various factors like severe immunosuppression, chronic diseases, prematurity, exposure to broad spectrum antibiotics and empirical use of antifungal agents (Deorukhar et al., 2014).
Candida albicans has from the past remained the most important cause of candidiasis accounting for about $60-80 \%$ of the infections (Manjunath et al., 2012). However, Non-albicans Candida (NAC) species like Candida glabrata, Candida krusei and Candida tropicalis are emerging as important opportunistic pathogens and this transition has had a significant clinical impact as, though clinical manifestations of infection caused by NAC species and 
Candida albicans are indistinguishable, NAC species exhibit decreased susceptibility to commonly used antifungal agents (Deorukhar et al., 2014; Baradkar et al., 2010). Candida tropicalis, Candida krusei and Candida glabrata have been found to be 32-fold less susceptible to fluconazole than Candida albicans (Agarwal et al., 2011). There is growing evidence to suggest that increasing use of Azoles is responsible for the epidemiological shift from Candida albicans to NAC species (Jain et al., 2012). Characterization of Candida to species level helps to identify those strains which might be intrinsically resistant to some antifungal agents (Jain, et al., 2012).

Conventional Candida speciation methods like morphology on Corn meal agar, carbohydrate fermentation and assimilation tests are time consuming taking from 72 hours to 2 weeks and the procedures are labor intensive (Baradkar et al., 2010).

The need for rapid identification of Candida species and the difficulty in detecting mixed cultures of Candida on Sabouraud's Dextrose Agar, has led to the development of several media that differentiate yeast species based on colony colour (Agarwal, et al., 2011). These media contain chromogenic substrates that react with species specific enzymes secreted by various Candida species producing colonies with various pigmentations, allowing organisms to be identified to the species level by their colour and colony characteristics (Horvath, et al., 2003). The present study was conducted to evaluate the efficacy of one such chromogenic media, HiCrome Candida agar as an alternative to conventional methods for the identification of Candida species.

\section{Materials and Methods}

The present cross-sectional study was conducted in the department of Microbiology, Shridevi Institute of Medical Sciences and Research Hospital, Tumkur for duration of one year from January 2014 to December 2014. During this period, a total of 48 isolates of Candida species were isolated from various clinical samples like urine, pus, body fluids, vaginal swab, etc. The study was submitted and approved by the Institutional Ethical Committee. All specimens were processed according to standard microbiological procedures. The specimens were first stained by Gram stain (Figure 1) followed by inoculation onto blood agar, MacConkey agar and thioglycollate broth for exudates and body fluids. The isolates were identified based on Gram staining and classified Candida albicans or non-albicans Candida based on germ tube test (Figure 2). Candida albicans and Candida dubliniensis were differentiated based on growth at $45^{\circ} \mathrm{C}$ (Chander, 2009).

Species identification was done by morphology on corn meal agar (Figure 3) by Dalmau plate culture method read after 48 hours of room temperature incubation (Shettar, et al., 2012).

HiCrome agar was procured from Himedia and prepared as per manufacturer's instructions. Candida isolates were subcultured onto HiCrome agar and incubated at $37^{\circ} \mathrm{C}$ for upto 48 hours for optimal development of colour. Isolates forming light green colonies were identified as Candida albicans, blue to metallic blue Candida tropicalis, purple fuzzy colonies Candida krusei and cream coloured colonies as Candida glabrata (Figure 4).

\section{Results and Discussion}

A total of 48 Candida isolates were obtained during the study period. Majority of isolates were from patients aged between 61-70 
years followed by 41-50 and 51-60 years. Maximum isolates were from respiratory samples followed by urine (Table 1).

Non-albicans Candida accounted for $50 \%$ of the isolates. The most common species isolated was Candida albicans (50\%) followed by Candida tropicalis $(27.08 \%)$ (Table 1).

HiCrome agar showed good growth of all Candida isolates after 48 hours of incubation. It was capable in accurately identifying 45 of the 48 isolates. The findings on HiCrome agar were in agreement with the conventional methods for Candida albicans, Candida tropicalis and Candida krusei.

HiCrome agar showed cream coloured colonies in case of 07 isolates which were identified as Candida glabrata based on colony morphology and colour. Two of these isolates were identified by conventional methods as Candida parapsilosis and one was identified as Candida guillermondii (Table 2).

Emergence of Non-albicans candida species with decreased susceptibility to antifungal agents has necessitated the identification of Candida to the species level. The conventional methods like inoculation on corn meal agar, carbohydrate assimilation and fermentation are expensive, timeconsuming and require expertise. Hence most laboratories do not go beyond germ tube test and limit their diagnosis to Candida albicansor Non-albicans Candida (Mehta, et al., 2016).

This study was conducted to analyse the efficacy of HiCrome agar in the rapid identification of Candida species. 48 Candida isolates were obtained from various clinical samples during a period of one year.
Candida albicans has historically been the predominant ca

use of candidiasis (Horvath, et al., 2003). The most common species identified was Candida albicans accounting for $50 \%$ of the isolates in the present study which was in agreement with other similar studies (Manjunath, et al., 2012; Baradkar, et al., 2010; Usharani, et al., 2011).

Because of increasing complexity in management and disease profiles of patients, there has been a surge of infections due to yeast other than Candida albicans (Agarwal S., et al.,2011).This epidemiological shift was demonstrated by studies conducted by Jain, et al., 2012; Jaya, et al., 2013 and Agarwal S., et al., 2011, where Candida tropicalis was the most common species isolated.

In a study by Shivaprakash et al, Candida albicans accounted for only 3.4\% isolates highlighting that Candida species other than Candida albicans have emerged as a major cause of invasive Candida infections (Shivaprakasha, et al., 2007).

In the present study, Candida tropicalis accounted for $27.08 \%$ of the isolates followed by Candida glabrata and Candida krusei (8.33\% each), Candida parapsilosis $(4.17 \%)$ and Candida guillermondii $(2.08 \%)$.

Candida glabrata has emerged as an important opportunistic pathogen worldwide and in India, Candida tropicalis has emerged as the most common cause of nosocomial candidemia (Giri, et al., 2012).

Studies have shown decreased susceptibility of Candida tropicalis, Candida glabrata and Candida krusei to commonly used azole antifungal agents (Pahwa, et al. 2014). 
Table.1 Distribution of various Candida species identified by Cornmeal agar

\begin{tabular}{|c|c|c|c|c|c|c|c|c|}
\hline Sample & $\begin{array}{l}\text { Candida } \\
\text { albicans }\end{array}$ & $\begin{array}{c}\text { Candida } \\
\text { tropicalis }\end{array}$ & $\begin{array}{c}\text { Candida } \\
\text { krusei }\end{array}$ & $\begin{array}{l}\text { Candida } \\
\text { glabrata }\end{array}$ & $\begin{array}{c}\text { Candida } \\
\text { parapsilosis }\end{array}$ & $\begin{array}{c}\text { Candida } \\
\text { guillermondii }\end{array}$ & Total & $\begin{array}{l}\% \text { of } \\
\text { total }\end{array}$ \\
\hline $\begin{array}{l}\text { Respiratory } \\
\text { samples }\end{array}$ & 17 & 03 & 00 & 01 & 01 & 00 & 22 & 45.83 \\
\hline Urine & 02 & 07 & 02 & 01 & 01 & 01 & 14 & 29.17 \\
\hline Pus & 00 & 02 & 01 & 02 & 00 & 00 & 05 & 10.41 \\
\hline Stool & 02 & 01 & 00 & 00 & 00 & 00 & 03 & 6.25 \\
\hline Vaginal swab & 02 & 00 & 00 & 00 & 00 & 00 & 02 & 4.17 \\
\hline $\begin{array}{l}\text { Peritoneal } \\
\text { fluid }\end{array}$ & 01 & 00 & 01 & 00 & 00 & 00 & 02 & 4.17 \\
\hline Total & 24 & 13 & 04 & 04 & 02 & 01 & 48 & 100 \\
\hline$\%$ of total & 50 & 27.08 & 8.33 & 8.33 & 4.17 & 2.08 & 100 & \\
\hline
\end{tabular}

Table. 2 Comparison of species identification by conventional methods and HiCrome agar

\begin{tabular}{|l|c|c|l|}
\hline \multicolumn{1}{|c|}{ Candia species } & Conventional method & HiCrome agar & \multicolumn{1}{|c|}{ Colour of colony } \\
\hline Candida albicans & 24 & 24 & Light green \\
\hline Candida tropicalis & 13 & 13 & Blue to metallic blue \\
\hline Candida krusei & 04 & 04 & purple fuzzy \\
\hline Candida glabrata & 04 & 07 & White to cream \\
\hline Candida parapsilosis & 02 & 00 & Cream \\
\hline Candida guillermondii & 01 & 00 & Cream \\
\hline
\end{tabular}


Fig.1 Gram stain of sputum showing Gram positive oval budding yeast cells with pseudohyphae

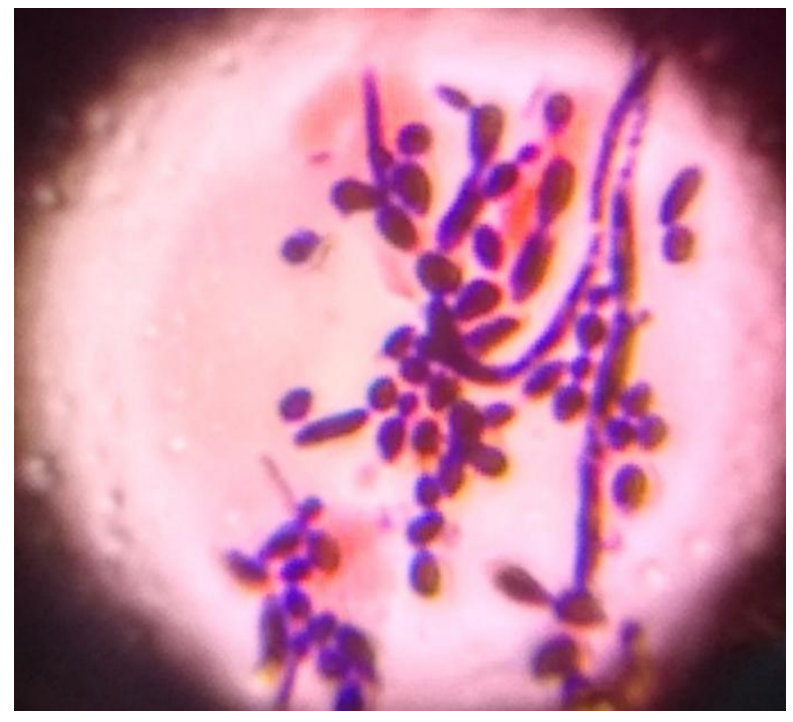

Fig.2 Germ tube formation by Candida albicans.

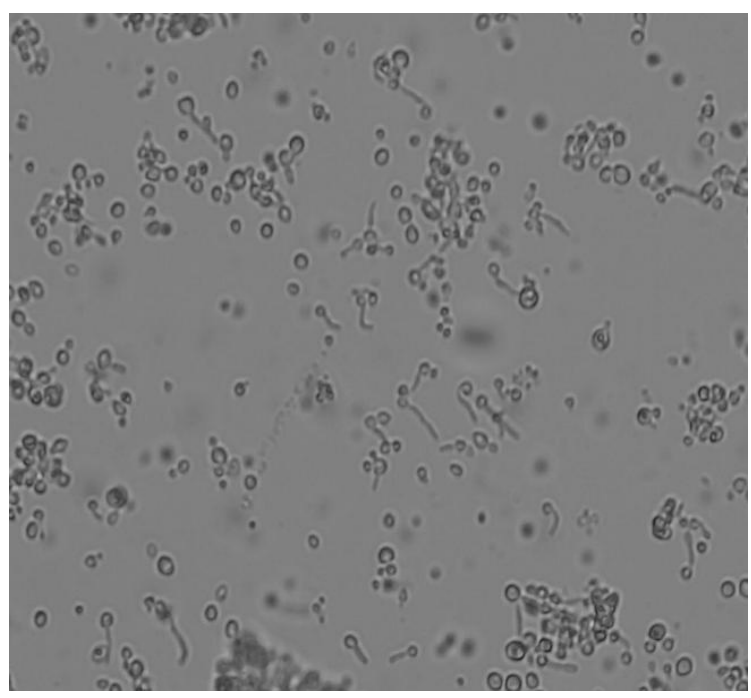


Fig.3 Morphology on Cornmeal agar: A - Candida albicans, B - Candida tropicalis, C - Candida glabrata, D - Candida parapsilosis, E-Candida krusei, F-Candida guillermondii.

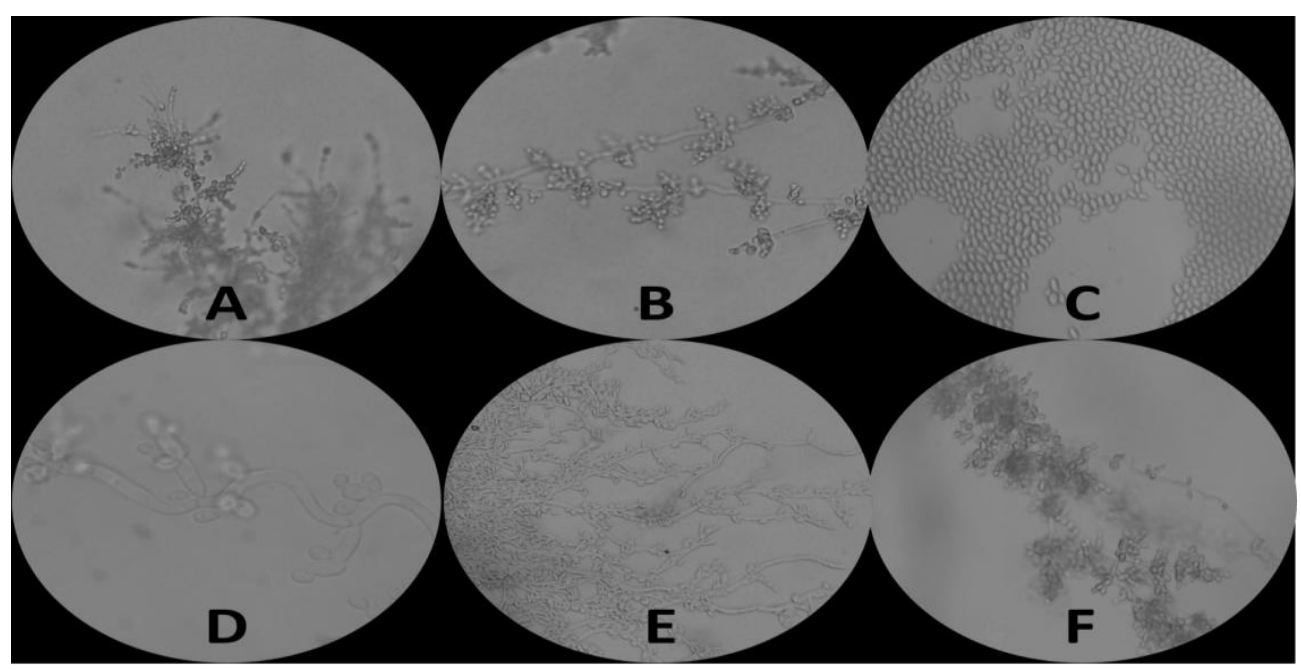

Fig.4 Colony morphology on HiCrome agar: Figure A-Candida tropicalis, Figure B - Candida albicans, Figure C - Candida glabrata, Figure D - Candida krusei.

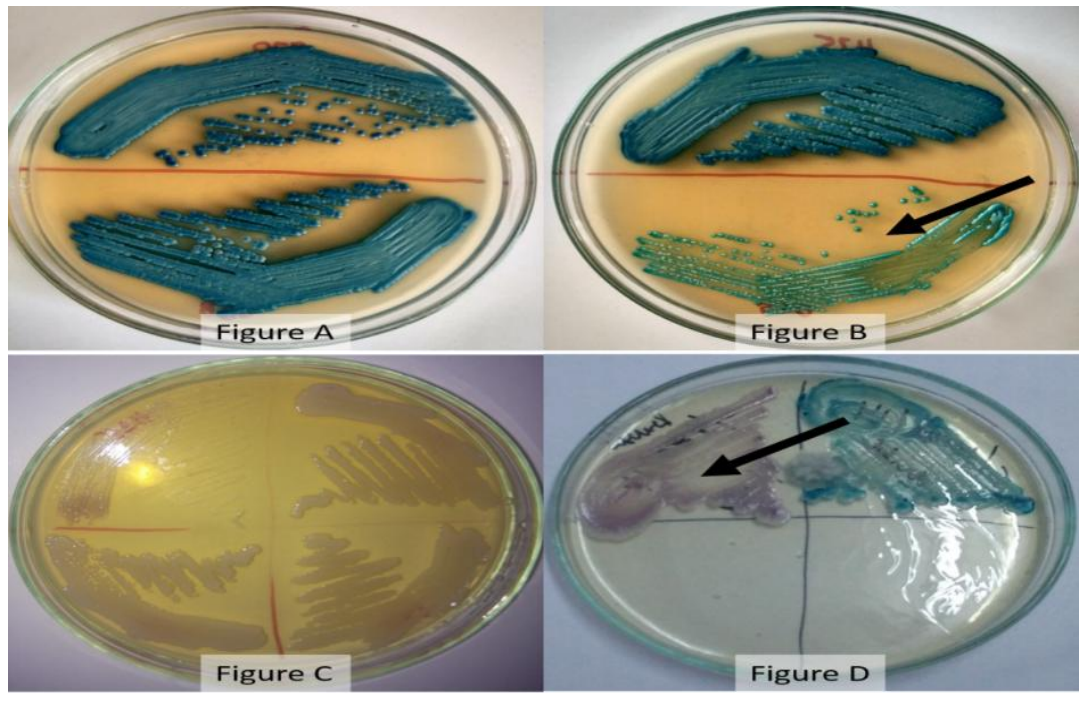


Given the potential for selection of these less-susceptible species by empirical antifungal treatment and prophylaxis, clinical laboratories should be able to identify these isolates to the species level (Agarwal, et al., 2011).

Several studies have been conducted in the last decade to assess the efficacy of HiCrome agar in identifying Candida species. Species identification by HiCrome agar were in agreement with those of conventional methods for Candida albicans, Candida tropicalis and Candida krusei. Our findings were similar to other studies conducted on HiCrome agar (Manjunath, et al., 2012; Shetttar, et al., 2012; Mehta, et al., 2016). All isolates of Candida albicansproduced light green colonies, Candida tropicalisblue to metallic blue colonies and Candida kruseiproduced purple fuzzy colonies showing $100 \%$ sensitivity and specificity for these isolates.

HiCrome agar misidentified three isolates as Candida glabrata based on colony colour and morphology. Two of these isolates were identified as Candida parapsilosis and one was identified as Candida guillermondii by conventional methods. The sensitivity and specificity of HiCrome agar in identification of Candida glabrata was $100 \%$ and $93.18 \%$ respectively. These findings were similar to other studieswhere Candida parapsilosis was misidentified as Candida glabrata due to similarity in colony morphology on HiCrome agar (Shettar, et al., 2012). But since Candida glabratadoes not produce pseudohyphae, they can be easily differentiated on corn meal agar. Hence use of HiCrome agar along with corn meal agar facilitates better identification of Candida species.

The present study emphasises the fact that HiCrome agar can be reliably used in the identification of various Candida species like Candida albicans, Candida tropicalis and Candida krusei. Other Candida species like Candida parapsilosis and Candida guillermondii produced similar colony morphology like Candida glabrata and lead to false identification of these species as Candida glabrata by HiCrome agar. In addition chromogenic medium also facilitates identification of different species of Candida in case of mixed infections (Agarwal, et al., 2011).

During the study period other important Candida species like Candida dubliniensis, Candida kefyr, etc. were not isolated. Hence the performance of HiCrome agar in identifying these isolates could not be assessed. A more extensive study with increased sample size would be required to assess the ability of HiCrome agar to isolate and identify these less commonly isolated Candida species.

Use of HiCrome agar allows for the rapid identification of Candida species allowing basic laboratories with limited resources to forgo tedious and time-consuming procedures like sugar assimilation tests. This facilitates rapid identification of especially those species like Candida krusei and Candida glabrata which have decreased susceptibility to commonly used anti-fungal agents and guides in the treatment of these infections.

In conclusion, the present study highlights the fact that use of HiCrome agar can be cost-effective alternative to the timeconsuming conventional methods in the rapid identification of Candida species reducing the time of identification from 1 week to 48 hours. The ease of preparing and using the medium, coupled with easy differentiation of Candida species based on colony colour enables basic clinical Microbiology laboratories to identify Candida to the species level. This will 
facilitate timely and appropriate initiation of anti-fungal therapy especially in patients suffering from systemic candidiasis.

\section{References}

Agarwal, S., Manchanda, V., Verma, N., Bhalla, P. 2011. Yeast identification in routine clinical microbiology laboratory and its clinical relevance. Indian J. Med. Microbiol., 29: 172-177.

Baradkar, V.P., Matur, M., Kumar, S. 2010. Hichrom Candida agar for identification of Candida species. Indian J. Pathol. Microbiol., 53(1): 93-95.

Chander, J. 2009. Textbook of Medical Mycology. $3^{\text {th }}$ edition. Mehta Publishers. New Delhi. Chapter 20: 266-83.

Deorukhkar, S.C., Saini, S. 2014. Laboratory approach for diagnosis of Candidiasis through ages. Int. J. Curr. Microbiol. App. Sci., 3(1): 206-218.

Giri, S., Kindo, A.J. 2012. A review of Candida species causing blood stream infection. Indian J. Med. Microbiol., 30(3): 270-278.

Horvath, L.L., Hosppenthal, D.R., Murray, C.K., Dooley, D.P. 2003. Direct isolation of Candida spp. from blood cultures on the chromogenic medium CHROMagar Candida. J. Clin. Microbiol., 41(6): 2626-2632.

Jain, N., Mathur, P., Misra, M.C., Behera, B., Xess, I., Sharma, S.P. 2012. Rapid identification of yeast isolates from clinical specimens in critically ill trauma patients. J. Lab. Physicians, 4(1): 30-34.

Jaya, S., Harita, V. 2013. Candida species isolated from various clinical samples and their susceptibility patterns to antifungals. J. Med. Microbiol. Infec., Dis., 1(1): 22-26.

Manjunath, V., Vidya, G.S., Sharma, A., Prakash, M.R., Murugesh. 2012. Speciation of Candida by Hicrome agar and sugar assimilation test in both HIV infected and non infected patients. Int. J. Biol. Med. Res., 3(2): 1778-1782.

Mehta, R., Myawahare, S.A. 2016. Evaluation of Hicrome Candida Differential Agar for species identification of Candida isolates from various clinical samples. Int. J. Contemporary Med. Res., 4(3): 1219-1222.

Pahwa, N., Kumar, R., Nirkhiwale, S., Bandi, A. 2014. Species distribution and drug susceptibility of Candida in clinical isolates from a tertiary care centre at Indore. Indian J. Med. Microbiol., 32(1): 44-48.

Shettar, S.K., Patil, A.B., Nadgir, S.D., Shepur, T.A., Mythri, B.A., Gadadavar, S. 2012. Evaluation of HiCrome differential agar for speciation of candida. J. Acad. Med. Sci., 2(3): 101104.

Shivaprakasha, S., Radhakrishnan, K., Karim, P.M.S. 2007. Candida spp. other than Candida albicans: A major cause of fungaemia in a tertiary care centre. Indian J. Med. Microbiol., 25(4): 405407.

Usharani, A., Bharathi, M., Sandhya, C., 2011. Isolation and characterisation of candida species from oropharyngeal secretions of HIV positive individuals. $N$. Dermatol. Online, 2(3): 119-124.

\section{How to cite this article:}

Soumya Kaup, Jaya Sankarankutty, H.V. Balasubrahmanya, Suma Kulkarni and M. Nirmala. 2016. Speciation of Candida using HiCrome Candida Differential Agar. Int.J.Curr.Microbiol.App.Sci. 5(7): 267-274. doi: http://dx.doi.org/10.20546/ijcmas.2016.507.027 